PROCEEDINGS OF THE

AMERICAN MATHEMATICAL SOCIETY

Volume 128, Number 11, Pages 3275-3283

S 0002-9939(00)05777-4

Article electronically published on April 28, 2000

\title{
FIXED POINT AND SELECTION THEOREMS IN HYPERCONVEX SPACES
}

\author{
M. A. KHAMSI, W. A. KIRK, AND CARLOS MARTINEZ YAÑEZ
}

(Communicated by David R. Larson)

\begin{abstract}
It is shown that a set-valued mapping $T^{*}$ of a hyperconvex metric space $M$ which takes values in the space of nonempty externally hyperconvex subsets of $M$ always has a lipschitzian single valued selection $T$ which satisfies $d(T(x), T(y)) \leq d_{H}\left(T^{*}(x), T^{*}(y)\right)$ for all $x, y \in M$. (Here $d_{H}$ denotes the usual Hausdorff distance.) This fact is used to show that the space of all bounded $\lambda$-lipschitzian self-mappings of $M$ is itself hyperconvex. Several related results are also obtained.
\end{abstract}

\section{INTRODUCTION}

Hyperconvex spaces are of interest for a number of reasons. In [1] it is shown that a metric space is hyperconvex if and only if it is injective. (A metric space $H$ is injective if given metric spaces $M$ and $N$ with $M$ a subspace of $N$ and a nonexpansive mapping $f: M \rightarrow H$, there exists a nonexpansive extension $\tilde{f}$ of $f$ such that $\tilde{f}: N \rightarrow H$.) Since it is known that every metric space has an injective hull [4], it follows that every metric space is isometric with a subspace of a (minimal) hyperconvex superspace. Also it is known that a real Banach space is hyperconvex if and only if it is isometrically isomorphic to a space of continuous real-valued functions defined on a stonian space. Thus hyperconvex Banach spaces include the $L_{\infty}$ spaces. (See, e.g., [3], 4], [5], 8] for classical results.)

This paper focuses on external hyperconvexity, a concept which was also introduced by Aronszajn and Panitchpakdi in their fundamental paper [1. Our main result, which extends the principal result of Sine 12, yields the fact that a lipschitzian set-valued mapping of a hyperconvex metric space into itself, taking externally hyperconvex values, always has a single valued selection which is lipschitzian for the same constant. This is used to show that the family of all bounded $\lambda$-lipschitzian mappings of a hyperconvex space into itself is itself hyperconvex. It

Received by the editors December 17, 1998.

1991 Mathematics Subject Classification. Primary 47H04, 47H10, 54H25; Secondary 47H09, $54 \mathrm{E} 40$.

Key words and phrases. Hyperconvex metric spaces, fixed points, selection theorems, fixed points.

This research was carried out while the first two authors were visiting the Universidad Catolica de Valparaiso on the occasion of the XXV Semana de la Matematica, October, 1998. They express their thanks to the sponsors for generous support and hospitality. The research of the third author was partially supported by FONDECYT grant no. 1980431.

(C)2000 American Mathematical Society 
is also shown that if the set-valued mapping is nonexpansive, then a nonexpansive selection exists which has precisely the same fixed point set. Several related intersection theorems and fixed point theorems are also obtained.

We begin by describing the relevant notation and terminology. For a subset $A$ of a metric space $M$ we use $N_{\varepsilon}(A)$ to denote the closed $\varepsilon$-neighborhood of $A$. Thus

$$
N_{\varepsilon}(A)=\{x \in M: \operatorname{dist}(x, A) \leq \varepsilon\} .
$$

An admissible subset of $M$ is a set of the form

$$
\bigcap_{i} B\left(x_{i} ; r_{i}\right)
$$

where $\left\{B\left(x_{i} ; r_{i}\right)\right\}$ is a family of closed balls centered at points $x_{i} \in M$ with respective radii $r_{i}$. The paper focuses on the following two fundamental concepts.

Definition 1. A metric space $M$ is said to be hyperconvex if given any family $\left\{x_{\alpha}\right\}$ of points of $M$ and any family $\left\{r_{\alpha}\right\}$ of real numbers satisfying

$$
d\left(x_{\alpha}, x_{\beta}\right) \leq r_{\alpha}+r_{\beta}
$$

it is the case that $\bigcap_{\alpha} B\left(x_{\alpha} ; r_{\alpha}\right) \neq \emptyset$.

Definition 2. A subset $E$ of a metric space $M$ is said to be externally hyperconvex (relative to $M$ ) if given any family $\left\{x_{\alpha}\right\}$ of points in $M$ and any family $\left\{r_{\alpha}\right\}$ of real numbers satisfying

$$
d\left(x_{\alpha}, x_{\beta}\right) \leq r_{\alpha}+r_{\beta} \text { and } \operatorname{dist}\left(x_{\alpha}, E\right) \leq r_{\alpha}
$$

it follows that $\bigcap_{\alpha} B\left(x_{\alpha} ; r_{\alpha}\right) \cap E \neq \emptyset$.

The fundamental result of [1] asserts that a metric space $M$ is hyperconvex if and only if it is injective. Thus $M$ is hyperconvex if given any two metric spaces $X$ and $Y$ with $Y$ a subspace of $X$, and any nonexpansive mapping $f: Y \rightarrow M$, then $f$ has a nonexpansive extension $\tilde{f}: X \rightarrow M$. Basic results about injective metric spaces can be found in [4]. (Also see [14].)

Regarding externally hyperconvex spaces, it is shown in [1] that any admissible subset of a hyperconvex space $M$ is externally hyperconvex relative to $M$, and that the externally hyperconvex subsets of $M$ are proximinal in $M$ (thus if $H$ is externally hyperconvex in $M$ and if $x \in M$, then there exists $h \in H$ such that $d(x, h)=\operatorname{dist}(x, H))$. This fact is used below in the proof of Theorem 1. (It is known [6] that a hyperconvex subset of $M$ need not be proximinal in $M$.)

In what follows we use $\mathcal{A}(M)$ to denote the family of all nonempty admissible subsets of $M$ and $\mathcal{E}(M)$ to denote the family of all nonempty bounded subsets of $M$ which are externally hyperconvex, in both instances endowed with the usual Hausdorff metric $d_{H}$. Recall that the distance between two closed subsets $A, B$ of a metric space in the Hausdorff sense is given by

$$
d_{H}(A, B)=\inf \left\{\varepsilon>0: A \subset N_{\varepsilon}(B) \text { and } B \subset N_{\varepsilon}(A)\right\} .
$$

It is well known (and easy to see) that an admissible subset of a hyperconvex space is itself hyperconvex. 


\section{MAIN RESUlts}

Our main result is the following selection theorem. Using a different method, Sine [12] (Theorem 1) obtained this result in the special case $T^{*}: H \rightarrow \mathcal{A}(H)$ with $T^{*}$ nonexpansive.

Theorem 1. Let $H$ be a hyperconvex metric space, let $S$ be any set, and let $T^{*}$ : $S \rightarrow \mathcal{E}(H)$. Then there exists a mapping $T: S \rightarrow H$ for which $T(x) \in T^{*}(x)$ for each $x \in S$ and for which $d(T(x), T(y)) \leq d_{H}\left(T^{*}(x), T^{*}(y)\right)$ for each $x, y \in S$.

Proof. Let $\mathfrak{F}$ denote the collection of all pairs $(D, T)$, where $D \subseteq S, T: D \rightarrow H$, $T(d) \in T^{*}(d) \forall d \in D$, and $d(T(x), T(y)) \leq d_{H}\left(T^{*}(x), T^{*}(y)\right)$ for each $x, y \in D$.

Notice that $\mathfrak{F} \neq \emptyset$ since $\left(\left\{x_{0}\right\}, T\right) \in \mathfrak{F}$ for any choice of $x_{0} \in S$ and $T\left(x_{0}\right) \in$ $T^{*}\left(x_{0}\right)$. Define an order relation on $\mathfrak{F}$ by setting

$$
\left(D_{1}, T_{1}\right) \preceq\left(D_{2}, T_{2}\right) \Leftrightarrow D_{1} \subset D_{2} \text { and }\left.T_{2}\right|_{D_{1}}=T_{1} .
$$

Let $\left\{\left(D_{\alpha}, T_{\alpha}\right)\right\}$ be an increasing chain in $(\mathfrak{F}, \preceq)$. Then it follows that $\left(\bigcup_{\alpha} D_{\alpha}, T\right) \in \mathfrak{F}$ where $\left.T\right|_{D_{\alpha}}=T_{\alpha}$. By Zorn's Lemma, $(\mathfrak{F}, \preceq)$ has a maximal element, say $(D, T)$. Assume $D \neq S$ and select $x_{0} \in S \backslash D$. Set $\tilde{D}=D \cup\left\{x_{0}\right\}$ and consider the set

$$
J=\bigcap_{x \in D} B\left(T(x) ; d_{H}\left(T^{*}(x), T^{*}\left(x_{0}\right)\right)\right) \cap T^{*}\left(x_{0}\right) .
$$

Since $T^{*}\left(x_{0}\right) \in \mathcal{E}(H)$ for each $x_{0} \in S, J \neq \emptyset \Leftrightarrow$ for each $x \in D$

$$
\operatorname{dist}\left(T(x), T^{*}\left(x_{0}\right)\right) \leq d_{H}\left(T^{*}(x), T^{*}\left(x_{0}\right)\right) .
$$

Also, since $T^{*}\left(x_{0}\right)$ is a proximinal subset of $H$, the above is true $\Leftrightarrow$ for each $x \in D$,

$$
B\left(T(x) ; d_{H}\left(T^{*}(x), T^{*}\left(x_{0}\right)\right)\right) \cap T^{*}\left(x_{0}\right) \neq \emptyset .
$$

By the definition of Hausdorff distance for each $\varepsilon>0$

$$
T^{*}(x) \subset N_{d_{H}\left(T^{*}(x), T^{*}\left(x_{0}\right)\right)+\varepsilon}\left(T^{*}\left(x_{0}\right)\right) .
$$

However by assumption $T(x) \in T^{*}(x)$, so it must be the case that for each $\varepsilon>0$,

$$
B\left(T(x) ; d_{H}\left(T^{*}(x), T^{*}\left(x_{0}\right)\right)+\varepsilon\right) \cap T^{*}\left(x_{0}\right) \neq \emptyset .
$$

Since $T^{*}\left(x_{0}\right)$ is proximinal in $H$, this in turn implies

$$
B\left(T(x) ; d_{H}\left(T^{*}(x), T^{*}\left(x_{0}\right)\right)\right) \cap T^{*}\left(x_{0}\right) \neq \emptyset .
$$

Thus we conclude $J \neq \emptyset$. Choose $y_{0} \in J$ and define

$$
\tilde{T}(x)=\left\{\begin{array}{cc}
y_{0} & \text { if } x=x_{0} \\
T(x) & \text { if } x \in D
\end{array}\right.
$$

Since

$$
d\left(\tilde{T}\left(x_{0}\right), \tilde{T}(x)\right)=d\left(y_{0}, T(x)\right) \leq d_{H}\left(T^{*}(x), T^{*}\left(x_{0}\right)\right),
$$

we conclude that $\left(D \cup\left\{x_{0}\right\}, \tilde{T}\right) \in \mathfrak{F}$, contradicting the maximality of $(D, T)$. Therefore $D=S$.

Corollary 1. Let $H$ be a hyperconvex metric space, let $(M, \rho)$ be a metric space, and suppose $T^{*}: M \rightarrow \mathcal{E}(H)$ is nonexpansive in the sense:

$$
d_{H}\left(T^{*}(x), T^{*}(y)\right) \leq \rho(x, y) \text { for each } x, y \in M .
$$

Then there exists a nonexpansive mapping $T: M \rightarrow H$ for which $T(x) \in T^{*}(x)$ for each $x \in M$. 
Proof. If $T^{*}$ is nonexpansive, then the selection $T$ assured by Theorem 1 is as well.

Corollary 2. Let $H$ be bounded and hyperconvex, and suppose $T^{*}: H \rightarrow \mathcal{E}(H)$ is nonexpansive. Then $T^{*}$ has a fixed point, that is, there exists $x \in H$ such that $x \in T^{*}(x)$.

Proof. The existence of a fixed point for the nonexpansive selection $T$ of $T^{*}$ follows from the well-known fixed point theorem of Sine [10] and Soardi [13].

In the following theorem $\operatorname{Fix}\left(T^{*}\right)=\left\{x \in H: x \in T^{*}(x)\right\}$. Corollary 2 implies $\operatorname{Fix}\left(T^{*}\right) \neq \emptyset$ if $H$ is bounded.

Theorem 2. Let $H$ be hyperconvex, let $T^{*}: H \rightarrow \mathcal{E}(H)$ be nonexpansive and suppose Fix $\left(T^{*}\right) \neq \emptyset$. Then there exists a nonexpansive mapping $T: H \rightarrow H$ with $T(x) \in T^{*}(x)$ for each $x \in H$ and for which Fix $(T)=$ Fix $\left(T^{*}\right)$.

Proof. Let $\mathfrak{F}$ denote the collection of all pairs $(D, T)$, where $D \supset$ Fix $\left(T^{*}\right), T: D \rightarrow$ $H, T(d) \in T^{*}(d)$ for all $d \in D, T(x)=x$ for all $x \in F i x\left(T^{*}\right)$, and $d(T(x), T(y)) \leq$ $d(x, y)$ for all $x, y \in D$. By assumption $\left(\operatorname{Fix}\left(T^{*}\right), I d\right) \in \mathfrak{F}$, so $\mathfrak{F} \neq \emptyset$. The argument is now a simple modification of the proof of Theorem 1 Define an order relation on $\mathfrak{F}$ by setting

$$
\left(D_{1}, T_{1}\right) \preceq\left(D_{2}, T_{2}\right) \Leftrightarrow D_{1} \subset D_{2} \text { and }\left.T_{2}\right|_{D_{1}}=T_{1} .
$$

Let $\left\{\left(D_{\alpha}, T_{\alpha}\right)\right\}$ be an increasing chain in $(\mathfrak{F}, \preceq)$. Then it follows that $\left(\bigcup_{\alpha} D_{\alpha}, T\right) \in \mathfrak{F}$ where $\left.T\right|_{D_{\alpha}}=T_{\alpha}$. By Zorn's Lemma, $(\mathfrak{F}, \preceq)$ has a maximal element, say $(D, T)$. Assume $D \neq H$ and select $x_{0} \in H \backslash D$. Set $\tilde{D}=D \cup\left\{x_{0}\right\}$ and consider the set

$$
J=\bigcap_{x \in D} B\left(T(x) ; d\left(x, x_{0}\right)\right) \cap T^{*}\left(x_{0}\right)
$$

Since $T^{*}\left(x_{0}\right) \in \mathcal{E}(H)$ for each $x_{0} \in H, J \neq \emptyset \Leftrightarrow$ for each $x \in D$,

$$
\operatorname{dist}\left(T(x), T^{*}\left(x_{0}\right)\right) \leq d\left(x, x_{0}\right) .
$$

Also, since $T^{*}\left(x_{0}\right)$ is a proximinal subset of $H$, the above is true $\Leftrightarrow$ for each $x \in D$,

$$
B\left(T(x) ; d\left(x, x_{0}\right)\right) \cap T^{*}\left(x_{0}\right) \neq \emptyset .
$$

Using the definition of Hausdorff distance and the fact that $T^{*}$ is nonexpansive, for each $\varepsilon>0$

$$
T^{*}(x) \subset N_{d_{H}\left(T^{*}(x), T^{*}\left(x_{0}\right)\right)+\varepsilon}\left(T^{*}\left(x_{0}\right)\right) \subset N_{d\left(x, x_{0}\right)+\varepsilon}\left(T^{*}\left(x_{0}\right)\right) .
$$

However by assumption $T(x) \in T^{*}(x)$, so it must be the case that for each $\varepsilon>0$,

$$
B\left(T(x) ; d\left(x, x_{0}\right)+\varepsilon\right) \cap T^{*}\left(x_{0}\right) \neq \emptyset .
$$

Since $T^{*}\left(x_{0}\right)$ is proximinal in $H$, this in turn implies

$$
B\left(T(x) ; d\left(x, x_{0}\right)\right) \cap T^{*}\left(x_{0}\right) \neq \emptyset .
$$

Thus we conclude $J \neq \emptyset$. Choose $y_{0} \in J$ and define

$$
\tilde{T}(x)=\left\{\begin{array}{cc}
y_{0} & \text { if } x=x_{0} \\
T(x) & \text { if } x \in D .
\end{array}\right.
$$

Since

$$
d\left(\tilde{T}\left(x_{0}\right), \tilde{T}(x)\right)=d\left(y_{0}, T(x)\right) \leq d\left(x, x_{0}\right),
$$


we conclude that $\left(D \cup\left\{x_{0}\right\}, \tilde{T}\right) \in \mathfrak{F}$, contradicting the maximality of $(D, T)$. Therefore $D=H$.

This in turn gives Corollary 3 of 12 .

Corollary 3. Let $H$ be hyperconvex, let $T^{*}: H \rightarrow \mathcal{E}(H)$ be nonexpansive, and suppose Fix $\left(T^{*}\right) \neq \emptyset$. Then Fix $\left(T^{*}\right)$ is hyperconvex.

Proof. The same conclusion holds for nonexpansive $T: H \rightarrow H$.

In view of Corollary $3 \mathrm{Fix}\left(T^{*}\right)$ is a nonexpansive retract of $H$, and an approach of Lin and Sine [9] can be used to show that a retraction $R$ of $H$ onto Fix $\left(T^{*}\right)$ exists which commutes with the selection $T$ of Theorem 2

Theorem 1 also yields a set-valued 'Schauder' theorem.

Corollary 4. Let $H$ be compact and hyperconvex, and suppose $T^{*}: H \rightarrow \mathcal{E}(H)$ is continuous. Then $T^{*}$ has a fixed point.

As another application of Theorem 1 we show that the family of all bounded $\lambda$-lipschitzian functions of a hyperconvex space $M$ into itself is itself hyperconvex. For two such functions we define distance in the usual way, that is, if $f, g: M \rightarrow M$, set

$$
d(f, g)=\sup _{x \in M} d(f(x), g(x))
$$

For this result we also need the following lemma due to R. Sine [11].

Lemma 1. If $M$ is hyperconvex and if $D=\bigcap_{\alpha} B\left(z_{\alpha} ; r_{\alpha}\right)$, then for any $\rho>0$

$$
N_{\rho}(D)=\bigcap_{\alpha} B\left(z_{\alpha} ; r_{\alpha}+\rho\right) \text {. }
$$

Theorem 3. Let $M$ be hyperconvex and for $\lambda>0$ let $\mathfrak{F}_{\lambda}$ denote the family of all bounded $\lambda$-lipschitzian functions of $M$ into $M$. Then $\mathfrak{F}_{\lambda}$ is itself a hyperconvex space.

Proof. Suppose $\left\{f_{\alpha}\right\} \subset \mathfrak{F}_{\lambda}$ and $\left\{r_{\alpha}\right\} \subset \mathbb{R}$ satisfy $d\left(f_{\alpha}, f_{\beta}\right) \leq r_{\alpha}+r_{\beta}$. Then for each $x \in M$

$$
d\left(f_{\alpha}(x), f_{\alpha}(x)\right) \leq r_{\alpha}+r_{\beta},
$$

so in view of the hyperconvexity of $M$

$$
J(x):=\bigcap_{\alpha} B\left(f_{\alpha}(x) ; r_{\alpha}\right) \neq \emptyset .
$$

We show that $d_{H}(J(x), J(y)) \leq \lambda d(x, y)$ for each $x, y \in M$. To see this it clearly suffices to show that for each $x, y \in M$

$$
J(x) \subset N_{\lambda d(x, y)}(J(y)) .
$$

However if $z \in J(x)$, then for each $\alpha$

$$
\begin{aligned}
d\left(z, f_{\alpha}(y)\right) & \leq d\left(z, f_{\alpha}(x)\right)+d\left(f_{\alpha}(x), f_{\alpha}(y)\right) \\
& \leq d\left(z, f_{\alpha}(x)\right)+\lambda d(x, y) \\
& \leq r_{\alpha}+\lambda d(x, y) .
\end{aligned}
$$

Using Sine's Lemma we now have

$$
z \in \bigcap_{\alpha} B\left(f_{\alpha}(y) ; r_{\alpha}+\lambda d(x, y)\right)=N_{\lambda d(x, y)}(J(y)) .
$$


In view of Theorem [1 it is possible to select $f(x) \in J(x)$ for each $x \in M$ so that $f \in \mathfrak{F}_{\lambda}$. Since $f \in \bigcap_{\alpha} B\left(f_{\alpha} ; r_{\alpha}\right), \mathfrak{F}_{\lambda}$ is hyperconvex.

This leads to the following.

Corollary 5. Let $M$ be a bounded hyperconvex metric space and let $f \in \mathfrak{F}_{1}$. Then the family

$$
R:=\left\{r \in \mathfrak{F}_{1}: r(M) \subset F i x(f)\right\}
$$

is a nonexpansive retract of $\mathfrak{F}_{1}$.

Proof. The mapping $T_{f}: \mathfrak{F}_{1} \rightarrow \mathfrak{F}_{1}$ defined via the formula $T_{f}(g)=f \circ g$ is nonexpansive and has a nonempty fixed point set which is a nonexpansive retract of $\mathfrak{F}_{1}$ ([2]). However, $r \in \operatorname{fix}\left(T_{f}\right) \Leftrightarrow r \in R$.

We conclude this section with the following observation.

Proposition 1. Let $M$ be a hyperconvex metric space and suppose $A$ is an externally hyperconvex subset $M$. Then $N_{\varepsilon}(A)$ is externally hyperconvex (in $M$ ) for each $\varepsilon>0$.

Proof. Let $\left\{x_{\alpha}\right\} \subset M$ and $\left\{r_{\alpha}\right\} \subset \mathbb{R}$ satisfy $d\left(x_{\alpha}, x_{\beta}\right) \leq r_{\alpha}+r_{\beta}$ and $\operatorname{dist}\left(x_{\alpha}, N_{\varepsilon}(A)\right)$ $\leq r_{\alpha}$. The latter inequality implies $\operatorname{dist}\left(x_{\alpha}, A\right) \leq r_{\alpha}+\varepsilon$. Since $A$ is externally hyperconvex, this in turn implies

$$
A \cap\left(\bigcap_{\alpha} B\left(x_{\alpha} ; r_{\alpha}+\varepsilon\right)\right) \neq \emptyset .
$$

By Sine's Lemma

$$
\bigcap_{\alpha} B\left(x_{\alpha} ; r_{\alpha}+\varepsilon\right)=N_{\varepsilon}\left(\bigcap_{\alpha} B\left(x_{\alpha} ; r_{\alpha}\right)\right)
$$

thus

$$
A \cap N_{\varepsilon}\left(\bigcap_{\alpha} B\left(x_{\alpha} ; r_{\alpha}\right)\right) \neq \emptyset .
$$

Therefore

$$
N_{\varepsilon}(A) \cap\left(\bigcap_{\alpha} B\left(x_{\alpha} ; r_{\alpha}\right) \neq \emptyset\right.
$$

and we conclude that $N_{\varepsilon}(A)$ is externally hyperconvex in $M$.

\section{Hyperconvex intersections}

While the intersection of two admissible subsets of a given hyperconvex space is again admissible, in general it is not the case that the intersection of two hyperconvex subspaces of a hyperconvex space is itself hyperconvex, even if one of them is admissible. However the following is true.

Lemma 2. Let $H$ be a hyperconvex metric space. Suppose $E \subset H$ is externally hyperconvex relative to $H$ and suppose $A$ is an admissible subset of $H$. Then $E \cap A$ is externally hyperconvex relative to $H$. 
Proof. Suppose $\left\{x_{\alpha}\right\}$ and $\left\{r_{\alpha}\right\}$ satisfy $d\left(x_{\alpha}, x_{\beta}\right) \leq r_{\alpha}+r_{\beta}$ and $\operatorname{dist}\left(x_{\alpha}, E \cap A\right) \leq r_{\alpha}$. Since $A$ is admissible, $A=\bigcap_{i \in I} B\left(x_{i} ; r_{i}\right)$, and since $B\left(x_{\alpha} ; r_{\alpha}\right) \cap A \neq \emptyset$, it follows that $d\left(x_{\alpha}, x_{i}\right) \leq r_{\alpha}+r_{i}$ for each $i \in I$. Also, since $A \subset B\left(x_{i} ; r_{i}\right)$, it follows that $\operatorname{dist}\left(x_{i}, E \cap A\right) \leq r_{i}$ and that $d\left(x_{i}, x_{j}\right) \leq r_{i}+r_{j}$ for each $i, j \in I$. Therefore by external hyperconvexity of $E$

$$
\left(\bigcap_{i} B\left(x_{i} ; r_{i}\right)\right) \cap\left(\bigcap_{\alpha} B\left(x_{\alpha} ; r_{\alpha}\right)\right) \cap E=\bigcap_{\alpha} B\left(x_{\alpha} ; r_{\alpha}\right) \cap(A \cap E) \neq \emptyset .
$$

This leads to the following.

Theorem 4. Let $\left\{H_{i}\right\}$ be a descending chain of nonempty externally hyperconvex subsets of a bounded hyperconvex space $H$. Then $\bigcap_{i} H_{i}$ is nonempty and externally hyperconvex in $H$.

Proof. A result of Baillon 2] assures that $D:=\bigcap_{i} H_{i} \neq \emptyset$. To see that $D$ is externally hyperconvex let $\left\{x_{\alpha}\right\} \subset H$ and $\left\{r_{\alpha}\right\} \subset \mathbb{R}$ satisfy $d\left(x_{\alpha}, x_{\beta}\right) \leq r_{\alpha}+r_{\beta}$ and $\operatorname{dist}\left(x_{\alpha}, D\right) \leq r_{\alpha}$. Since $H$ is hyperconvex, we know that $A:=\bigcap_{\alpha} B\left(x_{\alpha} ; r_{\alpha}\right) \neq \emptyset$. Also, since $\operatorname{dist}\left(x_{\alpha}, D\right) \leq r_{\alpha}$, we have $\operatorname{dist}\left(x_{\alpha}, H_{i}\right) \leq r_{\alpha}$ for each $i$; so by external hyperconvexity of $H_{i}$ we conclude $A \cap H_{i} \neq \emptyset$ for each $i$. By Lemma $2\left\{A \cap H_{i}\right\}$ is a descending chain of nonempty hyperconvex subsets of $H$, so again by [2] $\bigcap_{i}\left(A \cap H_{i}\right)=A \cap D \neq \emptyset$.

Another consequence of Lemma 2 provides yet further evidence of the ubiquitous nature of hyperconvexity.

Theorem 5. Let $H$ be a hyperconvex metric space and suppose $T^{*}: H \rightarrow \mathcal{E}(H)$. Then the family $S\left(T^{*}\right)$, consisting of all mappings $T: H \rightarrow H$ for which $T(x) \in$ $T^{*}(x)$ and $d(T(x), T(y)) \leq d_{H}\left(T^{*}(x), T^{*}(y)\right)$ for each $x, y \in H$, is hyperconvex.

Proof. Suppose $\left\{T_{\alpha}\right\} \subset S\left(T^{*}\right)$ and $\left\{r_{\alpha}\right\}$ satisfy $d\left(T_{\alpha}, T_{\beta}\right) \leq r_{\alpha}+r_{\beta}$. Then since $T^{*}(x)$ is hyperconvex,

$$
J(x):=\left(\cap_{\alpha} B\left(T_{\alpha}(x) ; r_{\alpha}\right)\right) \cap T^{*}(x) \neq \emptyset
$$

for each $x \in H$. Moreover, by Lemma $2, J(x) \in \mathcal{E}(H)$. Therefore by Theorem 1 the mapping $x \longmapsto J(x)$ has a selection $T$ which satisfies $d(T(x), T(y))$ $\leq d_{H}\left(T^{*}(x), T^{*}(y)\right)$ for each $x, y \in H$. Thus $T \in \bigcap_{\alpha} B\left(T_{\alpha} ; r_{\alpha}\right) \cap S\left(T^{*}\right)$.

In [7] it is shown that a complete metric space $M$ is hyperconvex and has unique metric segments if and only if it is an $\mathbb{R}$-tree. Using this it is easy to see that the intersection of two hyperconvex subspaces of a hyperconvex space is always hyperconvex if the underlying space has unique metric segments. This fact yields the following result.

Theorem 6. Suppose $M$ is a hyperconvex metric space which has unique metric segments, and suppose $H$ is a bounded hyperconvex subspace of $M$. Then any nonexpansive mapping $T: H \rightarrow M$ which satisfies

$$
\inf \{d(x, T(x)): x \in H\}=0
$$

always has a fixed point. 
Proof. Since $M$ is hyperconvex, $T$ has a nonexpansive extension $\tilde{T}: M \rightarrow M$. Let

$$
F_{n}:=\{x \in M: d(x, \tilde{T}(x)) \leq 1 / n\}, n=1,2, \cdots .
$$

It is known [1] that each of the sets $F_{n}$ is a hyperconvex subspace of $M$, and clearly $H_{n}:=F_{n} \cap H \neq \emptyset$ for each $n$. Since $H$ has unique metric segments, $H_{n}$ is hyperconvex, so $\bigcap_{n=1}^{\infty} H_{n} \neq \emptyset([2])$. Clearly each point of $\bigcap_{n=1}^{\infty} H_{n}$ is a fixed point of $T$.

We conclude this section with a simple observation about admissible sets which is another easy consequence of Sine's Lemma.

Proposition 2. Suppose $M$ is hyperconvex, and let $U=\bigcap_{i \in I} B\left(x_{i} ; r_{i}\right)$ and $V=$ $\bigcap_{i \in I} B\left(y_{i} ; r_{i}\right)$. Then

$$
d_{H}(U, V) \leq \sup \left\{d\left(x_{i}, y_{i}\right): i \in I\right\} .
$$

Proof. Let $\rho:=\sup \left\{d\left(x_{i}, y_{i}\right): i \in I\right\}$ and let $x \in U$. Then

$$
d\left(x, y_{i}\right) \leq d\left(x, x_{i}\right)+d\left(x_{i}, y_{i}\right) \leq r_{i}+\rho .
$$

Thus

$$
x \in \bigcap_{i} B\left(y_{i} ; r_{i}+\rho\right)=N_{\rho}\left(\bigcap_{i} B\left(y_{i} ; r_{i}\right)\right)=N_{\rho}(V)
$$

and we conclude $U \subset N_{\rho}(V)$. Reversing the roles of $U$ and $V$ gives the conclusion.

\section{ExAmples}

It might be interesting to note that Theorem [6 fails without the assumption of unique metric segments, even if $H$ is an admissible set.

Example 1. Let $B$ denote the unit ball in $\ell_{\infty}$ and let $H=B\left(\mathbf{z}_{1} ; 1\right) \cap B\left(\mathbf{z}_{2} ; 1\right)$ where

$$
\mathbf{z}_{1}=(1,0,0, \cdots) \text { and } \mathbf{z}_{2}=(-1,0,0, \cdots) .
$$

Let $\mathbf{x}=\left(0, x_{2}, x_{3}, \cdots\right) \in H$ and define $T: H \rightarrow B$ by the formula

$$
T(\mathbf{x})=\left(1-\sup _{2 \leq i<\infty}\left(1-\frac{1}{i}\right)\left|x_{i}\right|,\left(1-\frac{1}{2}\right) x_{2}, \cdots,\left(1-\frac{1}{n}\right) x_{n}, \cdots\right) .
$$

Then $T$ is both nonexpansive and fixed point free. However, if $\mathbf{e}^{n}$ denotes the standard unit vector basis, then

$$
\left\|\mathbf{e}^{n}-T\left(\mathbf{e}^{n}\right)\right\|=\frac{1}{n}, \quad n=2,3, \cdots .
$$

Several facts about externally hyperconvex subsets can easily be deduced from simple examples in $\mathbb{R}_{\infty}^{2}$. As we mentioned earlier, it is shown in 1 that every admissible subset of a hyperconvex space is externally hyperconvex. It is not difficult to show that an externally hyperconvex subset of $\mathbb{R}_{\infty}^{2}$ is necessarily an admissible subset of $\mathbb{R}_{\infty}^{2}$. However it is easy to see that an externally hyperconvex subset of a hyperconvex space need not always be admissible.

Example 2. Let $H$ be the solid rectangle in $\mathbb{R}_{\infty}^{2}$ with corners $( \pm 2, \pm 1)$, and let $E$ be the line interval $[-2,2]$. Then $E$ is externally hyperconvex relative to $H$ but $E$ is not an admissible subset of $H$ (although clearly $E$ is an admissible subset of $\left.\mathbb{R}_{\infty}^{2}\right)$. Notice that $H$ itself is hyperconvex because it is an admissible subset of the hyperconvex space $\mathbb{R}_{\infty}^{2}$. 
The following example exhibits another curious property of external hyperconvexity.

Example 3. Let $H=\{(x, x): 0 \leq x \leq 1\}$. Then $H$ is not externally hyperconvex relative to $\mathbb{R}_{\infty}^{2}$. On the other hand, $H$ is externally hyperconvex relative to $H \cup$ $\{(1,0)\}$.

\section{REFERENCES}

[1] Aronszajn, N., and Panitchpakdi, P., Extensions of uniformly continuous transformations and hyperconvex metric spaces, Pacific J. Math. 6(1956), 405-439. MR 18:917c correction MR 19:1069h

[2] Baillon, J. B., Nonexpansive mappings and hyperconvex spaces, Contemp. Math. 72(1988), 11-19. MR 89k:54068]

[3] Herrlich, H., Hyperconvex hulls of metric spaces, Topology Appl. 44(1992), 181-187. MR 93f: 54043

[4] Isbell, J. R., Six theorems about injective metric spaces, Comment. Math. Helvetici 39(1964), 65-76. MR 32:431

[5] Isbell, J. R., Injective envelopes of of Banach spaces are rigidly attached, Bull. Amer. Math. Soc. 70(1984), 727-729. MR 32:1537

[6] Khamsi, M. A., Lin, M., and Sine, R., On the fixed points of commuting nonexpansive maps in hyperconvex spaces, J. Math. Anal. Appl. 168(1992), 372-380. MR 93j:47080

[7] Kirk, W. A., Hyperconvexity of $\mathbb{R}$-trees, Fund. Math. 156(1998), 67-72. MR 98k:54060

[8] Lacey, H. E., The Isometric Theory of Classical Banach Spaces, Springer-Verlag, New York, Heidelberg, Berlin, 1974. MR 58:12308

[9] Lin, M., and Sine, R., On the fixed point set of order preserving maps, Math. Zeit. 203(1990), 227-234. MR 91a:47074

[10] Sine, R., On nonlinear contraction semigroups in sup norm spaces, Nonlinear Analysis Theory, Methods \& Applications 3(1979), 885-890. MR 80i:47082

[11] Sine, R., Hyperconvexity and approximate fixed points, Nonlinear Analysis - Theory, Methods \& Applications 13(1989), 863-869. MR 90g:54041

[12] Sine, R., Hyperconvexity and nonexpansive multifunctions, Trans. Amer. Math. Soc. 315(1989), 755-767. MR 90a:54054

[13] Soardi, P., Existence of fixed points of nonexpansive mappings in certain Banach lattices, Proc. Amer. Math. Soc. 73(1979), 25-29. MR 80c:47051

[14] Sullivan, F., Ordering and completeness of metric spaces, Nieuw Arch. Wisk. 29(1981), 178193. MR 84d:54079

Department of Mathematical Sciences, University of Texas at El Paso, El Paso, TEXas 79968-0514

E-mail address: mohamed@math.utep.edu

Department of Mathematics, The University of Iowa, Iowa City, Iowa 52242-1419

E-mail address: kirk@math.uiowa.edu

Institute of Mathematics, Universidad Catolica de Valparaiso, Valparaiso, Chile

E-mail address: cmartine@ucv.cl 